\title{
Authentication of Processed Epimedii folium by EA-IRMS
}

\author{
Fengyan He, Mengyi Li, Yi He $\mathbb{D}$, Zhe Dong, Jin Cao, Zhong Dai, and Shuangcheng Ma $\mathbb{D}$ \\ National Institutes for Food and Drug Control, Beijing 100050, China \\ Correspondence should be addressed to Yi He; zjsheyi@sina.com and Shuangcheng Ma; masc@nifdc.org.cn
}

Received 6 November 2019; Accepted 12 December 2019; Published 3 February 2020

Academic Editor: Boryana M. Nikolova-Damyanova

Copyright $\odot 2020$ Fengyan He et al. This is an open access article distributed under the Creative Commons Attribution License, which permits unrestricted use, distribution, and reproduction in any medium, provided the original work is properly cited.

Processing of crude drug is a key character of traditional Chinese medicine (TCM), which could enhance the efficacy and/or reduce the toxicity of crude drugs to fulfill different requirements of TCM clinical practice. Epimedii folium (EF) is a widely used TCM. As a traditional method of TCM, EF is processed with refined mutton fat before being used in clinical practice. It has been reported that processing EF with mutton fat could improve the bioavailability and intestinal absorption of epimedium flavonoids and thus enhances the pharmacological effects. For economic benefits, it is possible to adulterate processed EF with unprocessed drug or process EF with cheaper plant oils. In the present study, 17 batches of crude and processed EF samples were collected from the Chinese market and 10 batches of replica processed drugs were prepared with different edible plant oils and animal fats in our laboratory. Elemental analyzer coupled with isotopic ratio mass spectrometry (EA-IRMS) was applied to determine the $\delta^{13} \mathrm{C}$ values of the cyclohexane extracts of those samples. Significant differences could be observed in the results. EA-IRMS could be used to discriminate raw EF, processed EF, and EF processed with $\mathrm{C} 3$ plant oils.

\section{Introduction}

As a widely used traditional Chinese medicine (TCM), Epimedii folium (EF) is the dried leaves of Epimedium brevicomu Maxim, E. sagittatum (Sieb. et Zucc.), E. pubescens Maxim, or E. koreanum Nakai [1]. EF was first recorded in Shen Nong Ben Cao Jing, an ancient book of TCM written about 2000 years ago [2]. In China, EF has been used as a tonic, antirheumatic, and aphrodisiac for thousands of years. Phytochemical and pharmacological investigations revealed that flavonoids were the main bioactive constituents of this drug [3-6].

Processing of crude drug is an important character of TCM [7]. Based on clinical practice and TCM theory, the processing could enhance the efficacy and/or reduce the toxicity of crude drugs. The processing method of EF listed on Chinese Pharmacopoeia was stir-frying with refined mutton fat. For each $100 \mathrm{~kg}$ EF, $20 \mathrm{~kg}$ refined mutton fat was used during the processing [1]. This method was first recorded in an ancient classic of TCM processing named Lei Gong Pao Zhi Lun (about 500 AD). Refined mutton fat was the fried fat of Capra hircus Linnaeus or Ovis aries Linnaeus. Refined mutton fat is "warm" and possesses the effects of "restoring deficiency," "moistening dryness," "dispelling wind," and "removing toxin," according to TCM theories [2]. Although raw and processed EF both have "antirheumatic" and "bone-strengthening" effects, it is believed that the processed product has better effect on "tonifying kidney." Modern pharmacological investigation has demonstrated that processing with refined mutton fat could improve the bioavailability and intestinal absorption of epimedium flavonoids and enhance the pharmacological effects of EF [8-11]. Therefore, stir-frying with refined mutton fat was essential for the clinical usage of EF.

Refined mutton fat was also a commonly used edible fat in Chinese diet. Its price was higher than normal plant oils such as rape oil and soybean oil. Economic benefit makes it possible to adulterate processed EF with raw materials or process $\mathrm{EF}$ with cheaper plant oils. In consideration of the improved pharmaceutical activities of processed EF, it is necessary to establish a reliable method to discriminate raw, processed drugs and drugs processed with other plant oils.

In Chinese Pharmacopeia, the olfactory sensation of refined mutton fat was used for the authentication of processed EF [1]. No other method has been reported so far. Branched chain fatty acids (BCFAs) were considered as main 
determinants of the odor of refined mutton fat. Several methods have been reported for the determination of these components by gas chromatography (GC) or gas chromatography coupled with mass spectrometry (GC-MS) $[12,13]$. BCFAs were of relative volatility and in low concentration levels in many animal fats and could be influenced by age, gender, breed, and nutrition of animals [14]. Furthermore, the BCFAs might evaporate during mutton fat refining, drug processing, transport, and storage of processed EF. Thus, BCFAs were not ideal markers for the authentication of processed EF.

Isotopic ratio mass spectrometry (IRMS) is a special mass spectrometry technique which measures the relative abundance of isotopes of light elements [15]. Elemental analyzer coupled with IRMS (EA-IRMS) is a generally used IRMS interface which determines the average isotopic signal of the entire sample. Compared with other IRMS interfaces such as gas chromatography/combustion coupled with IRMS (GC/C/IRMS), EA-IRMS is fast and simple, without complicated sample pretreatment. EA-IRMS has been applied in the authenticity evaluation and geographical traceability of herbal drugs and foods especially in the characterization of plant oils and animal fats [16-19]. It has been reported that edible vegetable oil and animal fat possessed different $\delta^{13} \mathrm{C}$ values [18, 19]. Because the refined mutton fat is the main part of the nonpolar extract of processed $\mathrm{EF}$, it is possible that the nonpolar extracts of the raw and processed $\mathrm{EF}$ have different $\delta^{13} \mathrm{C}$ values. In the present study, 17 batches of raw and processed EF were collected, 1 batch of EF raw material was processed with different vegetable oils and animal fats in our laboratory, and the $\delta^{13} \mathrm{C}$ values of those samples were analyzed by EA-IRMS to discriminate raw and processed EF and the oils used for the processing of EF.

\section{Materials and Methods}

2.1. Sample Collection. Seventeen batches of EF samples were collected from market, including 8 batches of raw materials (No. 1-8) and 9 batches (No. 9-17) of processed drugs. In those samples, Sample Nos. 2, 3, and 7 were identified to be the leaves of Epimedium koreanum Nakai, and the rest samples were the leaves of E. brevicomu Maxim by Prof. Nanping Zhang (National Institutes for Food and Drug Control, Beijing, China).

2.2. Preparation of Replica Processed Drugs. To evaluate the influence of adjuvants on the $\delta^{13} \mathrm{C}$ value of processed $\mathrm{EF}$, raw material No. 6 was processed with different plant oils and animal fats in our laboratory to obtain samples R1R10. Plant oils included C3 plant oils (soybean oil (Soy), sunflower oil (Sun), rapeseed oil (Rap), and peanut oil (Pea)) and C4 plant oil (maize oil (Mai)). Three batches of mutton fats were used and labeled as Mut A, Mut B, and Mut C. As common edible fats, pork fat (Por) and beef fat (Bee) were chosen to be the possible adulterations of mutton fat. Plant oils were purchased from a local supermarket. Animal fats were fried with animal adipose tissues in our laboratory. The processing followed the method of Chinese pharmacopeia.

2.3. Extraction with Cyclohexane. The samples (raw materials, processed drugs, and replica processed drugs) were pulverized to powder. Then, $1 \mathrm{~g}$ of the drug powder was ultrasonically extracted with cyclohexane (analytical grade, Sinopharm Chemical Reagent Co. Ltd, Beijing, China) under ice bath for $30 \mathrm{~min}$. After filtration, the extract was dried under reduced pressure at $40^{\circ} \mathrm{C}$. The residuals were stored in fridge at $4^{\circ} \mathrm{C}$ before use.

2.4. $\delta^{13} \mathrm{C}$ Determination. The $\delta^{13} \mathrm{C}$ values of extracts and bulk adjuvant were measured using Thermo Flash 2000-HT elemental analyzer (Thermo Fisher Scientific, Bremen, Germany) coupled with a Thermo Delta-V Advantage isotope ratio mass spectrometer (Thermo Fisher Scientific) via a Thermo DELTA/MAT253 ConFlo IV Interface (Thermo Fisher Scientific). The elemental analyzer was controlled by Finnigan Eager 300 Isodat software (version 3.0). The oxidation and reduction reactors were heated to $960^{\circ} \mathrm{C}$, respectively. The oven temperature was set at $50^{\circ} \mathrm{C}$. The carrier gas $(\mathrm{He})$ flow was about $90 \mathrm{~mL} / \mathrm{min}$. The flow rate of $\mathrm{O}_{2}$ gas flash combustion was $180 \mathrm{~mL} / \mathrm{min}$. The run time of the experiment was approximately $5 \mathrm{~min}$ for a single run.

The carbon isotopic composition was expressed as the difference $(\delta)$ between an analyte and an international standard, Vienna Pee Dee Belemnite (VPDB), based on the following equation:

$$
\delta^{13} \mathrm{C}=\left(\frac{R_{\text {sample }}}{R_{\text {standard }}}-1\right) \times 1000,
$$

where $R_{\text {sample }}$ is the isotope ratio measured for the sample and $R_{\text {standard }}$ is the isotope ratio of international standard. The delta values are expressed in units "per mil" (\%o). Analysis of each sample was performed in triplicate. The isotopic values were calculated against international reference material urea (IVA33802174, $\delta^{13} \mathrm{C}=-43.26 \%$, IVA analysentechnik E. K., Germany). For calibration, the reference material was analyzed every 10 samples. As precision of analysis, the standard deviation (SD) of calculated urea results was below $0.15 \%$ and $0.2 \%$ for sample repeats.

2.5. Statistical Analysis. One-way ANOVA was performed on the cyclohexane extract amounts of drug samples. Independent samples $T$ test was conducted on $\delta^{13} \mathrm{C}$ values of cyclohexane extracts from raw and processed drugs. Pearson correlation analysis was performed on $\delta^{13} \mathrm{C}$ values of cyclohexane extracts of replica processed drugs and corresponding adjuvants. SPSS software (version 16.0) was used.

\section{Results and Discussion}

3.1. Yields of Cyclohexane Extracts. The yields of cyclohexane extracts of 8 batches of EF raw materials and 9 batches of purchased processed products ranged from 0.31 to $1.11 \%$ and 9.04 to $32.26 \%$, respectively (Table S1). For replica 


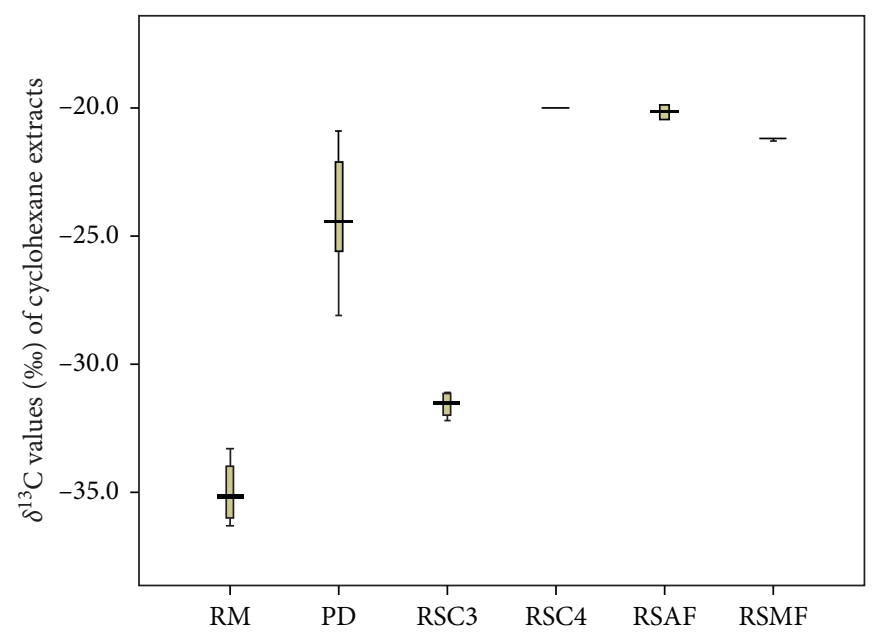

Figure 1: Boxplot of $\delta^{13} \mathrm{C}$ values of cyclohexane extracts from raw materials (RM), processed drugs (PD), replica samples processed with C3 plant oils (RSC3), replica samples processed with C4 plant oils (RSC4), replica samples processed with other animal fats (RSAF), and replica samples processed with mutton fats (RSMF).

processed drugs, the yields ranged from 10.22 to $15.21 \%$ (Table S2). It suggested that the nonpolar component amounts of processed products, either purchased from the Chinese market or reproduced in our laboratory, were significantly higher than that of the raw materials (one-way ANOVA, $p<0.05)$. The difference between cyclohexane extracts amounts of processed drugs and the replica processed drugs were not significant (one-way ANOVA, $p>0.05)$. Therefore, the nonpolar component amount was influenced greatly by the processing procedure and could be used as a marker for discrimination of raw and processed EF.

Compared with replica processed drugs, the nonpolar component amounts of purchased processed drugs ranged greatly. This might be attributed to the variety of specific processing parameters in different processing factories and probably heterogeneous distribution of refined mutton fat in bulk drugs.

\section{2. $\delta^{13} \mathrm{C}$ Determination of Cyclohexane Extracts of Collected} Samples. The $\delta^{13} \mathrm{C}$ values of 8 batches of raw materials and 9 batches of processed drugs ranged from -36.3 to $-33.3 \%$ and from -28.1 to $-20.9 \%$ (Table S1). As shown in Figure 1, the $\delta^{13} \mathrm{C}$ values of processed drugs are significantly higher than those of raw materials (independent samples $T$-test, $p<0.05) . \delta^{13} \mathrm{C}$ value of cyclohexane extract could be used as a key marker to distinguish EF raw materials and processed drugs.

3.3. $\delta^{13} C$ Determination of Adjuvants and Cyclohexane Extracts of Replica Processed Samples. Plant oils of Pea, Soy, Sun, and Rap possess $\delta^{13} \mathrm{C}$ values ranging from -31.4 to $-30.9 \%$, while Mai shows the $\delta^{13} \mathrm{C}$ value of $-17.5 \%$ o (Table S2). The results were consistent with other reports about the $\delta^{13} \mathrm{C}$ values of C3 and C4 plants oils $[19,20]$.

For animal fats, Por had the highest $\delta^{13} \mathrm{C}$ value of $-17.4 \%$, while Bee and Mut A, B, and C had the $\delta^{13} \mathrm{C}$ values nearly equal to $-19.0 \%$ o $(-19.0,-19.2,-18.9$, and $-19.6 \%$, Table S2).

The $\delta^{13} \mathrm{C}$ value of replica processed drugs made with Pea, Soy, Sun, and Rap oils ranged from -32.2 to $-31.1 \%$, and the $\delta^{13} \mathrm{C}$ value of those processed with Mai and 5 animal fats ranged from -21.3 to $-19.9 \%$ (Table S2).

Pearson correlation analysis revealed that $\delta^{13} \mathrm{C}$ values of adjuvants were significantly correlated to $\delta^{13} \mathrm{C}$ values of cyclohexane extracts of replica processed drugs (coefficient $=0.998$ ), which revealed that oil or fat used during processing dominated the $\delta^{13} \mathrm{C}$ value of cyclohexane extract for processed drugs.

It is well known that plants can be divided into C3 and $\mathrm{C} 4$ plants based on the difference of photosynthetic pathways. Most of the plants were C3 plants, expecting about 8100 species including the food crops maize, sugar cane, millet, and sorghum [21, 22]. C4 plants have higher natural ${ }^{13} \mathrm{C}$ enrichment than $\mathrm{C} 3$ plants. This is the reason why Mai shows the more positive $\delta^{13} \mathrm{C}$ value than other $\mathrm{C} 3$ plant oils.

The $\delta^{13} \mathrm{C}$ values of plant oils were mainly dominated by photosynthetic pathways and were relatively constant. For the adulterant processed with $\mathrm{C} 3$ plant oils, the $\delta^{13} \mathrm{C}$ value could be used as a prominent marker. But for adulterant processed with $\mathrm{C} 4$ plant oils (such as maize oil), blend oils including C3 and C4 oils, or other common edible animal fats, the current method was not adequate. It should be further studied.

3.4. $\delta^{13} \mathrm{C}$ Values of EF Processed with Animal Fats. In China, Sheep and goats were raised in a large scale including west plateaus, east, and middle plains [23]. It has been revealed that $\delta^{13} \mathrm{C}$ values of animal tissues were close related to the feed regime [24-26]. Feeding regimes of sheep and goat were different from place to place. More pastures would be feed in the pastoral area, and more crop by-products would be used in the crop area. Besides, silage, straw, concentrate feed, bran, soybean cake, cottonseed cake, and other feed were 
also generally used. Difference between feed regimes for sheep and goats should be the dominant factor for the varieties of the $\delta^{13} \mathrm{C}$ value of cyclohexane extracts of purchased processed drugs. Only the livestock feed with natural pastures (mostly C3 plants) could possess $\delta^{13} \mathrm{C}$ values close to $\mathrm{EF}$ raw materials [25]. However, in view of the high farming cost, the fats of pasture-fed livestock were less likely to be used as processing adjuvant. This assumption was also confirmed by the detected $\delta^{13} \mathrm{C}$ values of processed $\mathrm{EFs}$ (Table S1).

It should been noticed that maize (maize, silage maize, maize straw, or concentrated feed made with maize) was widely used in livestock production in China. As shown in Table S2, all drugs processed with animal fats show the $\delta^{13} \mathrm{C}$ values between $\mathrm{C} 3$ and $\mathrm{C} 4$ plant oils. A maize-based diet for different livestock also leaded to similar $\delta^{13} \mathrm{C}$ values of different animal fats. Thus EA-IRMS is not adequate for the discrimination of drugs processed with other animal fats.

On the other hand, the prices of pork, beef, and other animal fats were close to refined mutton fat, making the adulteration with animal fats less profitable than plant oils. Therefore, discrimination of the EF processed with other animal fats is not so urgent.

3.5. Optional Methods for the Authentication of Processed EF. According to our experiment results, together with reported traditional morphological characters, 3 methods could be suggested for the authentication of processed EF.

The first method is the morphological identification. According to Chinese Pharmacopeia, the processed EF should have a pale yellow surface with fatty luster and smells a slight odor of refined mutton fat, in contrast to the raw drug which is yellowish-green or greyish-green and with slight odor [1]. The changes in the morphological characters were the results of the used fat and the high temperature during processing. However, due to the diversities of mutton fat quality and processing parameters, the morphological identification should be based on personal professional experiences.

The amount of cyclohexane extract could also be used for the authentication of processed EF. As shown in Table S1, the cyclohexane extract amount ranged from $9.04 \%$ to $32.26 \%$ for processed drugs and 0.31 to $1.11 \%$ for raw materials. The difference between the cyclohexane extract amounts of raw and processed EFs was significant $(p<0.01)$ and could be used for the identification of raw and processed EF. Because of the added fats, the cyclohexane extract amount was increased after processing. Nevertheless, if plant oils were used during the processing, the cyclohexane extract amount could also be increased (Table S2).

The third authentication method is the $\delta^{13} \mathrm{C}$ value of cyclohexane extract. For processed EF, most of the cyclohexane extracts were the nonpolar components from the oil or fat used during processing. Thus, its $\delta^{13} \mathrm{C}$ value was corresponding to the used oil or fat, which was consistent with our study (Table S2). The different $\delta^{13} \mathrm{C}$ values of $\mathrm{C} 3$ plant oils and animal fats could be used to estimate the excipients used for EF processing.

\section{Conclusion}

In the present study, EA-IRMS was used for discrimination of raw, processed $\mathrm{EF}$, and the oil used during processing. Cyclohexane extract amount and its $\delta^{13} \mathrm{C}$ values were significantly different between the raw and processed EF. The drugs processed with $\mathrm{C} 4$ plant oils or other animal fats show the similar $\delta^{13} \mathrm{C}$ values with the drug processed with refined mutton fat. However, the drugs processed with $\mathrm{C} 3$ plant oils possess more negative $\delta^{13} \mathrm{C}$ values than the drugs processed with refined mutton fat, as described in Chinese Pharmacopeia. EA-IRMS could be applied to discriminate raw EF, processed $\mathrm{EF}$, and the $\mathrm{C} 3$ plant oils used during $\mathrm{EF}$ processing. The EA-IRMS methods have been established for the authenticity evaluation and geographical traceability of foods, including plant oils and edible animal fats [18-20]. However, few papers have reported about the application of EA-IRMS on the authentication of processed Chinese material medica. The application of EA-IRMS on the quality control of traditional Chinese medicine possesses unique advantages and should be paid more attention.

\section{Data Availability}

The data used to support the findings of this study are included within the article.

\section{Conflicts of Interest}

The authors declare that there are no conflicts of interest regarding the publication of this paper.

\section{Authors' Contributions}

Fengyan He and Mengyi Li equally contributed to this work.

\section{Supplementary Materials}

Table S1: amounts and $\delta^{13} \mathrm{C}$ values (\%) of cyclohexane extracts from 17 batches of collected samples $(n=3)$. Table S2: amounts and $\delta^{13} \mathrm{C}$ values (\%) of cyclohexane extracts from 10 replica processed drugs and $\delta^{13} \mathrm{C}$ values (\%) of corresponding adjuvant $(n=3)$. (Supplementary Materials)

\section{References}

[1] State Pharmacopoeia Committee, Pharmacopoeia of People's Republic of China, Volume I, China Medical Science and Technology Press, Beijing, China, 2015.

[2] Editorial Board of China Herbal, State Administration of Traditional Chinese Medicine, China Herbal, Book Three, Shanghai Science and Technology Publishers, Shanghai, China, 1999.

[3] J. F. Zhang, G. Li, C. L. Meng et al., "Total flavonoids of Herba Epimedii improves osteogenesis and inhibits osteoclastogenesis of human mesenchymal stem cells," Phytomedicine, vol. 16, no. 6-7, pp. 521-529, 2009.

[4] D. Zhang, J. Zhang, C. Fong, X. Yao, and M. Yang, "Herba epimedii flavonoids suppress osteoclastic differentiation and bone resorption by inducing G2/M arrest and apoptosis," Biochimie, vol. 94, no. 12, pp. 2514-2522, 2012. 
[5] H.-H. Xiao, C.-Y. Fung, S.-K. Mok et al., "Flavonoids from Herba epimedii selectively activate estrogen receptor alpha $(\mathrm{ER} \alpha)$ and stimulate ER-dependent osteoblastic functions in UMR-106 cells," The Journal of Steroid Biochemistry and Molecular Biology, vol. 143, pp. 141-151, 2014.

[6] W.-F. Chen, S.-K. Mok, X.-L. Wang et al., "Total flavonoid fraction of the Herba epimedii extract suppresses urinary calcium excretion and improves bone properties in ovariectomised mice," British Journal of Nutrition, vol. 105, no. 2, pp. 180-189, 2011.

[7] Z. Zhao, Z. Liang, K. Chan et al., "A unique issue in the standardization of Chinese Materia Medica: processing," Planta Medica, vol. 76, no. 17, pp. 1975-1986, 2010.

[8] J. Hou, J. Li, E. Sun, C. Zhu, and X. Jia, "Processing study of Epimedium flavonoids self-assembled micelles fried with suet oil on its bioavailability," Chinese Pharmaceutical Journal, vol. 52, no. 9, pp. 726-730, 2017.

[9] L. Cui, E. Sun, Q. Qian, H. Fan, J. Zou, and X. Jia, "Comparative study on effect of crude and different processed products of epimedium on pharmacokinetics characteristics in mice," China Journal of Chinese Materia Medica, vol. 38, no. 10, pp. 1614-1617, 2013.

[10] J. Li, E. Sun, X. Tan, and X. Jia, "Promotion of suet oil to absorption and transportation of total flavonoids from Epimedii Folium," Chinese Traditional and Herbal Drugs, vol. 46, no. 16, pp. 2439-2444, 2015.

[11] L. Cui, E. Sun, Z.-H. Zhang et al., "Enhancement of epimedium fried with suet oil based on in vivo formation of selfassembled flavonoid compound nanomicelles," Molecules, vol. 17, no. 11, pp. 12984-12996, 2012.

[12] E. Wong, C. B. Johnson, and L. N. Nixon, "The contribution of 4-methyloctanoic (hircinoic) acid to mutton and goat meat flavour," New Zealand Journal of Agricultural Research, vol. 18, no. 3, pp. 261-266, 1975.

[13] C. B. Johnson, E. Wong, E. J. Birch, and R. W. Purchas, "Analysis of 4-methyloctanoic acid and other medium chainlength fatty acid constituents of ovine tissue lipids," Lipids, vol. 12, no. 4, pp. 340-347, 1977.

[14] P. J. Watkins, G. Rose, L. Salvatore et al., "Age and nutrition influence the concentrations of three branched chain fatty acids in sheep fat from Australian abattoirs," Meat Science, vol. 86, no. 3, pp. 594-599, 2010.

[15] Y. He, J. Wang, M. Wang, and J. Zhang, "Discrimination of wild and domestic deer musk using isotope ratio mass spectrometry," Journal of Mass Spectrometry, vol. 53, no. 11, pp. 1078-1085, 2018.

[16] A. Simsek, M. Bilsel, and A. C. Goren, "13C/12C pattern of honey from Turkey and determination of adulteration in commercially available honey samples using EA-IRMS," Food Chemistry, vol. 130, no. 4, pp. 1115-1121, 2012.

[17] Z. Tian, S. Du, C. Liu et al., "Identification of geographical origins of raw American ginseng and tablets based on stable isotope ratios," Journal of Chromatography B, vol. 1009-1010, pp. 73-79, 2016.

[18] N. N. Ahmad Nizar, J. M. Nazrim Marikkar, and D. M. Hashim, "Differentiation of lard, chicken fat, beef fat and mutton fat by GCMS and EA-IRMS techniques," Journal of Oleo Science, vol. 62, no. 7, pp. 459-464, 2013.

[19] L.-X. Guo, X.-M. Xu, J.-P. Yuan, C.-F. Wu, and J.-H. Wang, "Characterization and authentication of significant Chinese edible oilseed oils by stable carbon isotope analysis," Journal of the American Oil Chemists' Society, vol. 87, no. 8, pp. 839-848, 2010.
[20] H. Lu, C. Wei, H. Fu, X. Li, Q. Zhang, and J. Wang, "Identification of recycled cooking oil and edible oils by iodine determination and carbon isotopic analysis," Journal of the American Oil Chemists Society, vol. 92, no. 11-12, pp. 1549-1553, 2015.

[21] R. F. Sage, "A portrait of the C4 photosynthetic family on the 50th anniversary of its discovery: species number, evolutionary lineages, and Hall of Fame," Journal of Experimental Botany, vol. 67, no. 14, pp. 4039-4056, 2016.

[22] X.-G. Zhu, S. P. Long, and D. R. Ort, "What is the maximum efficiency with which photosynthesis can convert solar energy into biomass?," Current Opinion in Biotechnology, vol. 19, no. 2, pp. 153-159, 2008.

[23] National Development and Reform Commission, "National development plan for beef and mutton production (20132020)," National Development and Reform Commission, Beijing, China, 2019, http://zfxxgk.ndrc.gov.cn/web/iteminfo. jsp?id=286.

[24] B. Bahar, F. J. Monahan, A. P. Moloney, P. O’Kiely, C. M. Scrimgeour, and O. Schmidt, "Alteration of the carbon and nitrogen stable isotope composition of beef by substitution of grass silage with maize silage," Rapid Communications in Mass Spectrometry, vol. 19, no. 14, pp. 1937-1942, 2005.

[25] E. Piasentier, R. Valusso, F. Camin, and G. Versini, "Stable isotope ratio analysis for authentication of lamb meat," Meat Science, vol. 64, no. 3, pp. 239-247, 2003.

[26] H. C. Norman, M. G. Wilmot, D. T. Thomas, D. G. Master, and D. K. Revell, "Stable carbon isotopes accurately predict diet selection by sheep fed mixtures of C3 annual pastures and saltbush or C4 perennial grasses," Livestock Science, vol. 121, no. 2-3, pp. 162-172, 2009. 